\title{
Nombramiento de un representante vital: actitud del paciente y del personal de enfermería
}

\author{
Núria Pujolar Fulquet, Rosario Castillo Rosa, Alicia Noguera Llauradó, Laia Cabrera de las Heras, Mª José \\ Soler Romeo
}

Hospital del Mar. Barcelona

\section{Introducción:}

Debido a la problemática que se genera ante una situación de incapacidad del paciente para poder decidir por sí mismo cuando no puede expresar su voluntad y en ausencia de un Documento de Voluntades Anticipadas, la existencia de un interlocutor válido es de gran ayuda para el equipo asistencial. Este interlocutor, o representante vital, es la persona en la que el paciente deposita su confianza y a la que transmite sus voluntades con la finalidad de que sean respetadas en situaciones en las que él mismo no podrá manifestarlas. Desde el Comité de Ética Asistencial y del Servicio de Nefrología, se diseñó un proyecto para conocer la opinión de los pacientes acerca de la figura del representante vital y saber si estarían dispuestos a nombrar el suyo propio después de recibir la información necesaria sobre este tema por parte de enfermeras del Servicio.

\section{Objetivos:}

1. Conocer la actitud de los pacientes respecto a la figura del representante vital.

2. Estimar el número de pacientes dispuestos a nombrar un representante vital.

3. Conocer la opinión de las enfermeras del estudio.

\section{Material y métodos:}

Estudio observacional transversal exploratorio con pacientes nefrológicos que ingresaron en la Unidad de Hospitalización del Servicio de Nefrología entre marzo del 2012 y febrero del 2013. Se definieron criterios de exclusión y se elaboró un documento informativo para el paciente, un consentimiento informado de participación, un cuestionario para el paciente y otro para la enfermera. Mediante criterios consensuados, la enfermera informó al paciente sobre el representante vital, aclaró sus dudas, proporcionó el documento informativo y al día siguiente entregó y recogió el cuestionario cumplimentado. Se decidió limitar la inclusión de pacientes a las situaciones en las que estaba disponible una enfermera con el tiempo suficiente para completar el protocolo diseñado.

\section{Resultados:}

De los 335 pacientes diferentes que ingresaron a cargo de Nefrología en el periodo de estudio, se incluyeron un total de 100 pacientes, de los cuáles: $\bullet 64$ pacientes $(62,74 \%)$ mostraron interés, de ellos: 47 pacientes (47\%) nombraron representante vital, con una media de edad del 61,7.17 pacientes participaron activamente pero no nombraron representante vital -36 pacientes $(36 \%)$, no mostraron interés: 11 por diferencias culturales, 7 no quisieron ni oír hablar del tema y 18 han escuchado la explicación pero no quieren colaborar en el estudio. - El tiempo medio invertido en cada paciente ha sido de 20 minutos $\bullet$ La valoración de las enfermeras en cuanto a privacidad y entorno para tratar el tema ha sido de inadecuado en todos los casos.

\section{Conclusiones:}

- La aplicación del protocolo de nombramiento de representante vital nos ha permitido culminar el 
proceso con éxito en prácticamente la mitad de casos en los que se intenta.

- Con los recursos humanos disponibles, sólo se ha podido entrevistar a menos de un tercio de todos los pacientes ingresados.

- Dos tercios de los pacientes muestran interés por el proceso, por lo que una planificación adecuada podría permitir mejores resultados en el nombramiento de un representante vital.

\section{Referencias Bibliográficas}

1. Comitè de Bioètica de Catalunya. Consideracions sobre el document de voluntats anticipades. 2 ed. Barcelona: Direcció General de Recursos Sanitaris; 2010.

2. Solsona JF. Voluntades Anticipadas: una herramienta para anticipar acontecimientos y facilitar la asistencia urgente. Emergencias 2007;19:239-240.
3. Marcos del Cano A.M. Voluntades anticipadas. A: Elizari Basterra F.J.(Dir). 10 palabras clave ante el final de la vida. Estella: Verbo Divino; 2007.p.389426.

4. Solsona J.F, Sucarrats A, Maull E, Barbat C, García S, Villares S. Toma de decisiones al final de la vida. Encuesta sobre la actitud ante la muerte en el paciente crítico. Med. Clin. 2003;120(9):335-6.

5. Lotto L, Manfritani A, Rigoni D, Rumiati R, Sartori G, Birbaumer N. Attitudes Towards End-ofLife Decisions and the Subjective Concepts of Consciousness: An Empirical Analysis. PLoS ONE 2012;7(2):E31735.

6. McGowan CM. Legal aspects of end-of-life care. Crit Care Nurse 2011;31(5):64-9.

7. Sahuquillo, MR. Instrucciones para morir. Periódico" El Pais", Sociedad. Domingo 21 de abril 2013. Pag 38. 